\title{
Endoscopic and Clinicopathological Features of Superficial Non-Ampullary Duodenal Tumor Based on the Mucin Phenotypes
}

\author{
Yoichi Akazawa ${ }^{a}$ Hiroya Ueyama ${ }^{a}$ Sho Tsuyama ${ }^{b}$ Atsushi lkeda ${ }^{a}$ \\ Noboru Yatagai ${ }^{a}$ Hiroyuki Komori ${ }^{a}$ Tsutomu Takeda ${ }^{a}$ Kohei Matsumoto ${ }^{a}$ \\ Kenshi Matsumoto ${ }^{a}$ Takashi Hashimoto $^{c}$ Natsumi Tomita ${ }^{c}$

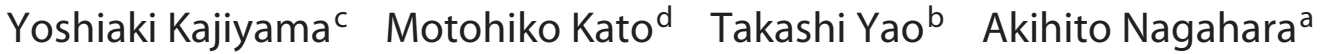 \\ ${ }^{a}$ Department of Gastroenterology, Juntendo University School of Medicine, Tokyo, Japan; ${ }^{b}$ Department of \\ Human Pathology, Juntendo University School of Medicine, Tokyo, Japan; 'Department of Esophageal and \\ Gastroenterological Surgery, Juntendo University School of Medicine, Tokyo, Japan; dDivision of Research and \\ Development for Minimally Invasive Treatment, Cancer Center, Keio University School of Medicine, Tokyo, Japan
}

\section{Keywords}

Non-ampullary duodenal tumor · Mucin phenotype ·

Magnifying endoscopy with narrow-band imaging

\begin{abstract}
Aims: We aimed to clarify the endoscopic/clinicopathological features of superficial non-ampullary duodenal epithelial tumors (SNADETs) based on their mucin phenotypes. Methods: We analyzed 62 SNADET lesions and classified them based on mucin phenotypic expression. Endoscopic and clinicopathological findings were compared according to mucin phenotypes. Results: Eleven lesions had the gastric phenotype (GP) and 43 lesions had the intestinal phenotype (IP). All GP lesions were located in the first portion of the duodenum, while most IP lesions (72.1\%) were located in the second portion $(p<0.01)$. Tumor size was significantly larger in the GP than in the IP group (14.4 mm vs. $10.2 \mathrm{~mm}, p<0.05$ ). Reddish color (72.7\% in GP vs. $37.2 \%$ in IP, $p<0.05)$, type $0-1$ (72.7\% vs. $11.6 \%, p<0.01)$, lobular/granular pattern $(81.8 \%$ vs. $4.7 \%, p<0.01)$, and category $4 / 5$ in Vienna classification (81.8\% vs. $30.2 \%, p<0.01)$ were observed significantly more often in the GP than in the IP group. Regarding findings of
\end{abstract}

$\begin{array}{ll}\text { karger@karger.com } & \begin{array}{l}\text { C } 2020 \text { The Author(s) } \\ \text { Pww.karger.com/dig }\end{array} \\ \text { Published by S. Karger AG, Basel } \\ \text { This article is licensed under the Creative Commons Attribution- } \\ \text { NonCommercial-NoDerivatives 4.0 International License (CC BY- } \\ \text { SC-ND) (http://www.karger.com/Services/OpenAccessLicense). } \\ \text { BoPEN ACCESS } & \begin{array}{l}\text { Usage and distribution for commercial purposes as well as any dis- } \\ \text { tribution of modified material requires written permission. }\end{array}\end{array}$

magnifying endoscopy with narrow-band imaging (M-NBI), white opaque substance $(22.2 \%$ in GP vs. $89.7 \%$ in IP, $p<$ $0.01)$ and light blue crest ( $0 \%$ vs. $43.6 \%, p<0.05)$ were significantly less frequently observed in the GP group. Ovalshaped marginal epithelium $(66.7 \%$ vs. $17.9 \%, p<0.01)$, dense pattern (55.6\% vs. $2.6 \%, p<0.01)$, and dilatation of the intervening part (100\% vs. $12.8 \%, p<0.01)$ were more frequently observed in the GP group. Conclusions: SNADETs showed distinct endoscopic/clinicopathological features according to the mucin phenotype. Tumor location, coloration, macroscopic type, and endoscopic findings including $\mathrm{M}-\mathrm{NBI}$ are useful to distinguish the mucin phenotypes of SNADETs.

(C) 2020 The Author(s) Published by S. Karger AG, Basel

\section{Introduction}

Although superficial non-ampullary duodenal epithelial tumors (SNADETs) are relatively rare [1], the detection of SNADETs has been increasing due to advances in recent endoscopic technology [2]. However, endoscopic resection of SNADETs is associated with a high risk for 
adverse events, such as perforation or bleeding due to anatomical reasons $[3,4]$. In addition, preoperative biopsy could lead to fibrosis of the submucosal layer, leading to difficulty in subsequent endoscopic therapy [5]. Thus, accurate preoperative diagnosis is important for SNADETs.

The association between the clinicopathological features of SNADETs and mucin phenotypes has been analyzed in several studies [6-9]. The results of these studies showed that SNADETs with the gastric phenotype (GP) had more aggressive biological behavior than those with the intestinal phenotype (IP). Therefore, if the mucin phenotype of SNADETs could be predicted, it would be helpful to consider the indication of endoscopic resection. Recently, the effectiveness of magnifying endoscopy for the preoperative diagnosis of SNADETs has been reported [10-14]. However, the association between mucin phenotypes and endoscopic findings in SNADETs has not been well elucidated.

In this study, we classified SNADETs histopathologically and aimed to clarify their endoscopic and clinicopathological features, including findings on conventional white-light imaging (WLI) and magnifying endoscopy with narrow-band imaging (M-NBI) based on their mucin phenotypes.

\section{Methods}

\section{Study Population}

We collected 65 lesions of SNADET that were resected by endoscopic procedures at our hospital between February 2013 and November 2017. Among them, 3 lesions that underwent insufficient histopathological evaluation due to damage to the specimen were excluded. The remaining 62 lesions were enrolled in this study, and mucin phenotypes of all lesions were assessed based on the criteria described below. Endoscopic and clinicopathological findings were compared between the mucin phenotypes.

\section{Histopathological and Immunohistochemical Assessment}

Four-micrometer-thick serial tissue sections prepared from formalin-fixed, paraffin-embedded tissues were subjected to hematoxylin and eosin (HE) staining and immunohistochemistry (IHC). In this study, the phenotypic expression of the tumors was evaluated by histopathological findings of both $\mathrm{HE}$ staining and IHC. The following antibodies were used for IHC: MUC5AC (NCL-MUC-5AC, Novocastra, Newcastle upon Tyne, UK), MUC6 (NCL-MUC-6), MUC2 (NCL-MUC-2), and CD10 (NCLCD10-270) using a Dako EnVision Kit. SNADETs were classified into 4 groups according to the mucin phenotype: the GP, the IP, the mixed phenotype (MP), and unclassified phenotype (UP). The GP was defined as tumors showing gastric mucosal differentiation (i.e., foveolar epithelium or gastric glands) on HE staining, and positivity for only MUC5AC and/or MUC6 on IHC. IP was defined as tumors showing intestinal mucosal differentiation (i.e., goblet cells, Paneth cells, and brush border) on HE staining, and positivity for only MUC2 and/or CD10 on IHC. These findings in more than $10 \%$ of the tumor area were considered as positive. Lesions showing both phenotypic expressions were classified into the MP group, and lesions with no phenotypic expression were classified into the UP group. Moreover, we histopathologically classified the lesions into category 3 (mucosal low-grade neoplasia) or category 4/5 (mucosal high-grade neoplasia/submucosal invasion by carcinoma) in accordance with the Vienna classification [15]. In all study patients, histopathological diagnoses were made by 2 pathologists specialized in gastrointestinal pathology (S.T. and T.Y.) at our hospital.

\section{Endoscopic and Clinical Assessment}

The endoscopic and clinicopathological characteristics that were examined were age, gender, operative method, tumor location, tumor size, coloration, macroscopic type, lobular/granular pattern, category of the Vienna classification, and M-NBI findings. If a tumor showed multiple colors or macroscopic types, the predominant color or macroscopic type was adopted, respectively. Macroscopic types were assessed according to the Paris endoscopic classification [16]. The lobular/granular pattern was defined as lobular or granular structure of the tumor on white-light endoscopy (WLI) (Fig. 1). As for M-NBI findings, the following factors were assessed according to previous studies: demarcation line (DL), microvascular pattern (MVP), microsurface pattern (MSP) $[17,18]$, light blue crest (LBC) [19], white opaque substance (WOS) [20], and dense pattern [21]. Additionally, we assessed 2 findings of M-NBI: dilatation of the intervening part (DIP), which reflects dilatation of the part between crypt openings, and ovalshaped marginal epithelium (OME), which reflects a villous or papillary glandular structure.

In all study patients, endoscopy was performed with the GIFH260Z or GIF-H290Z endoscope (Olympus Corporation, Tokyo, Japan) by 4 experienced endoscopists (K.M., H.U., H.K, and Y.A.) at our hospital. The video processor was set as follows: the structure enhancement function was set at the B6 level for WLI, and B8 for M-NBI, with the NBI color mode fixed at level 1. The 4 expert endoscopists retrospectively assessed the endoscopic images. If the diagnosis was different, the experts discussed it among each other to reach a consensus.

Statistical Analysis

All statistical analyses were performed with EZR (Saitama Medical Center, Jichi Medical University, Saitama, Japan) [22], which is a graphical user interface for $R$ (The $R$ Foundation for Statistical Computing, Vienna, Austria). Continuous data were compared with the Mann-Whitney $U$ test. Categorical analysis of variables was performed using the Fisher exact test. A $p$ value $<0.05$ was considered to indicate a statistically significant difference.

\section{Results}

\section{Clinicopathological Characteristics of All SNADETs}

A summary of the clinicopathological characteristics of all SNADETs is shown in Table 1 . There were 40 males and 22 females, with ages ranging from 41 to 89 years 
Fig. 1. Endoscopic findings of a SNADET with the GP. a, b WLI shows a reddish pedunculated lesion in the first portion of the duodenum. The lobular/granular pattern was positive. c, d M-NBI reveals a dense pattern and DIP (red arrow). On the other hand, WOS and light blue crest are both negative. SNADET, superficial non-ampullary duodenal epithelial tumor; GP, gastric phenotype; M-NBI, magnifying endoscopy with narrow-band imaging; WOS, white opaque substance; WLI, white-light imaging; DIP, dilatation of the intervening part.
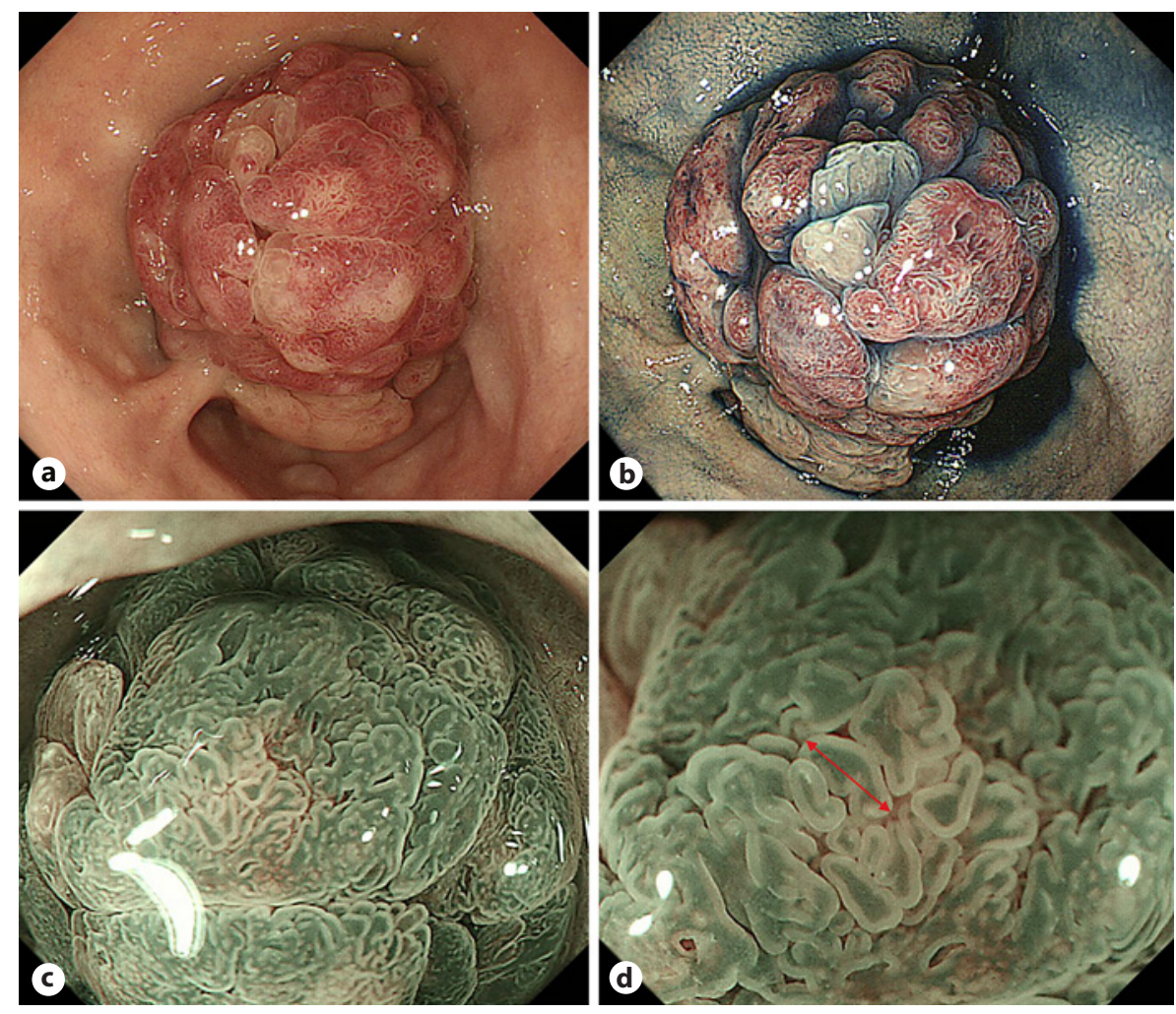

(mean 66.4 years). The lesions were resected by endoscopic mucosal resection for 43 lesions and by endoscopic submucosal dissection for 19 lesions. About half of the lesions were located in the second portion of the duodenum (first portion/second portion/third portion = 24/33/5). The mean tumor size was $12.7 \mathrm{~mm}$ (range: 2-35 $\mathrm{mm})$. The color of SNADETs was red/isochromatic or white in 30/32 lesions. Macroscopically, type 0-IIa was predominantly observed $(0-\mathrm{I} / 0$-IIa/0-IIc $=18 / 33 / 11)$. In the Vienna classification, there were 32 lesions in category 3 and 30 lesions in category 4 or 5 . As for the mucin phenotypes, there were 11 lesions with the GP, 43 lesions with the IP, 7 lesions with the MP, and 1 lesion with UP.

\section{Comparison of Clinicopathological Findings between the GP and IP Groups}

In the following analysis, we excluded lesions with the MP $(n=7)$ or UP $(n=1)$ to clarify the specific findings of lesions with the GP and lesions with the IP. The remaining 54 lesions were enrolled in the clinicopathological analysis. Clinicopathological findings of the GP and IP groups are shown in Table 2. All GP lesions were located in the first portion of the duodenum, while most IP lesions were located in the second portion of the duodenum
Table 1. Clinicopathological characteristics of all SNADETs $(n=$ 62)

\begin{tabular}{lc}
\hline Mean age, years [range] & $66.4[41-89]$ \\
Gender (male/female) & $40 / 22$ \\
Operative method (EMR/ESD) & $43 / 19$ \\
Tumor location (first/second/third portion) & $24 / 33 / 5$ \\
Mean tumor size, mm [range] & $12.7[2-35]$ \\
Coloration (red/isochromatic or white) & $30 / 32$ \\
Macroscopic type (0-I/0-IIa/0-IIc) & $18 / 33 / 11$ \\
Mucin phenotype (GP/IP/MP/UP) & $11 / 43 / 7 / 1$ \\
Vienna classification (category 3/category 4 or 5) & $32 / 30$ \\
\hline
\end{tabular}

SNADETs, superficial non-ampullary duodenal epithelial tumors; EMR, endoscopic mucosal resection; ESD, endoscopic submucosal dissection; GP, gastric phenotype; IP, intestinal phenotype; MP, mixed phenotype; UP, unclassified phenotype.

(first/second/third $=11 / 0 / 0$ in GP vs. 8/31/4 in IP, $p<$ $0.01)$. The mean tumor size was significantly larger in the GP group than in the IP group (14.4 $\mathrm{mm}$ [range: $7-25$ $\mathrm{mm}$ ] in GP vs. $10.2 \mathrm{~mm}$ [range: $2-30 \mathrm{~mm}$ ] in IP, $p<0.05)$. Reddish color $(72.7 \%, 8 / 11$ in GP vs. $37.2 \%, 16 / 43$ in IP, $p<0.05)$, type 0 -I $(72.7 \%, 8 / 11$ in GP vs. $11.6 \%, 5 / 43$ in IP, $p<0.01)$, lobular/granular pattern $(81.8 \%, 9 / 11$ in GP 
Table 2. Comparison of clinicopathological findings between the GP and IP groups $(n=54)$

\begin{tabular}{lccc}
\hline & GP $(n=11)$ & IP $(n=43)$ & $p$ value \\
\hline Mean age, years [range] & $67.3[48-89]$ & $65.2[41-83]$ & 0.72 \\
Gender (male/female) & $9 / 2$ & $26 / 17$ & 0.29 \\
Operative method (EMR/ESD) & $8 / 3$ & $30 / 13$ & 1.00 \\
Tumor location (first/second/third portion) & $11 / 0 / 0$ & $8 / 31 / 4$ & $<0.01$ \\
Mean tumor size, mm [range] & $14.4[7-25]$ & $10.2[2-30]$ & $<0.05$ \\
Coloration (red/isochromatic or white) & $8 / 3$ & $16 / 27$ & $<0.05$ \\
Macroscopic type (0-I/0-IIa/0-IIc) & $8 / 3 / 0$ & $5 / 28 / 10$ & $<0.01$ \\
Lobular/granular pattern (+/-) & $9 / 2$ & $2 / 41$ & $<0.01$ \\
Vienna classification (category 3/category 4 or 5) & $2 / 9$ & $30 / 13$ & $<0.01$ \\
\hline
\end{tabular}

GP, gastric phenotype; IP, intestinal phenotype; EMR, endoscopic mucosal resection; ESD, endoscopic submucosal dissection.

Table 3. Comparison of M-NBI findings between the GP and IP groups $(n=48)$

\begin{tabular}{lllc}
\hline & $\begin{array}{l}\text { GP } \\
(n=9)\end{array}$ & $\begin{array}{l}\text { IP } \\
(n=39)\end{array}$ & $p$ value \\
\hline DL (+/-) & $9 / 0$ & $39 / 0$ & NA \\
MVP (regular/irregular/absent) & $4 / 4 / 1$ & $11 / 10 / 18$ & 0.13 \\
MSP (regular/irregular/absent) & $3 / 6 / 0$ & $26 / 12 / 1$ & 0.10 \\
WOS (+/-) & $2 / 7$ & $35 / 4$ & $<0.01$ \\
LBC (+/-) & $0 / 9$ & $17 / 22$ & $<0.05$ \\
OME (+/-) & $6 / 3$ & $7 / 32$ & $<0.01$ \\
Dense pattern (+/-) & $5 / 4$ & $1 / 38$ & $<0.01$ \\
DIP (+/-) & $9 / 0$ & $5 / 34$ & $<0.01$ \\
\hline
\end{tabular}

GP, gastric phenotype; IP, intestinal phenotype; DL, demarcation line; MVP, microvascular pattern; MSP, microsurface pattern; WOS, white opaque substance; LBC, light blue crest; OME, ovalshaped marginal epithelium; DIP, dilatation of the intervening part; NA, not assessed.

vs. $4.7 \%, 2 / 43$ in IP, $p<0.01)$, and category $4 / 5$ in Vienna classification $(81.8 \%, 9 / 11$ in GP vs. $30.2 \%, 13 / 43$ in IP, $p<0.01$ ) were observed significantly more frequently in the GP than in the IP group. No significant differences were observed in age (67.3 years [range: $48-89$ years] in GP vs. 65.2 years [range: $41-83$ years] in IP, $p=0.72$ ), gender (male/female $=9 / 2$ in GP, 26/17 in IP, $p=0.29$ ), and operative method (EMR/ESD $=8 / 3$ in GP, 30/13 in IP, $p=1.00$ ) between the GP and IP groups.

\section{Comparison of M-NBI Findings between the GP and IP Groups}

The M-NBI findings of the GP and IP groups are shown in Table 3 ( $n=48,6$ lesions were excluded because of no picture with M-NBI). All lesions showed demarcation line clearly $(100 \%, 48 / 48)$. WOS $(22.2 \%, 2 / 9$ in GP vs. $89.7 \%$, $35 / 39$ in IP, $p<0.01)$ and LBC $(0 \%, 0 / 9$ in GP vs. $43.6 \%$, $17 / 39$ in IP, $p<0.05$ ) were significantly less frequently observed in the GP than in the IP group. OME $(66.7 \%, 6 / 9$ in GP vs. $17.9 \%, 7 / 39$ in IP, $p<0.01)$, dense pattern (55.6\%, $5 / 9$ in GP vs. $2.6 \%, 1 / 39$ in IP, $p<0.01)$, and DIP ( $100 \%$, $9 / 9$ in GP vs. $12.8 \%, 5 / 39$ in IP, $p<0.01)$ were significantly more frequently observed in the GP than in the IP group. There were no significant differences between the 2 groups with respect to MVP and MSP. Representative cases of the GP and IP groups are shown in Figures 1-4.

\section{Discussion}

From the results of this study, specific findings of the GP group were as follows: located in the first portion of the duodenum, reddish color, type 0-I, lobular/granular pattern, OME, dense pattern, DIP, and high frequency of category 4/5 in Vienna classification. Specific findings of the IP group were as follows: located in the second portion of the duodenum, isochromatic or whitish color, type 0 -IIa or 0-IIc, WOS, LBC, and high frequency of category 3 in Vienna classification (Fig. 5).

Previous reports showed that SNADETs of the GP frequently show macroscopically pedunculated type and are located in the first portion of the duodenum, similar to our results $[6,9,14,23]$. With regard to coloration, although Hijikata et al. [23] reported that there was no significant association between coloration and phenotypes, our results showed that isochromatic or white color was observed significantly more often in the IP than in the GP group. Among the 48 lesions in which M-NBI was per- 

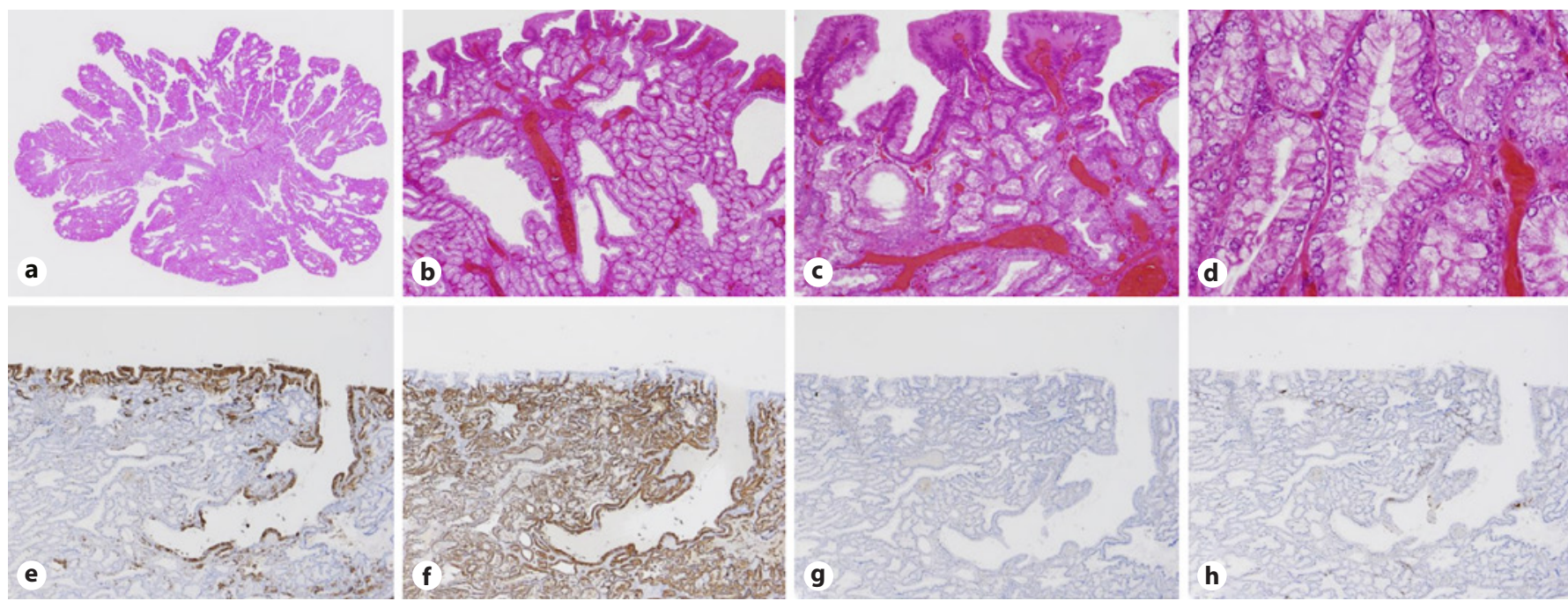

Fig. 2. Histopathological findings of a SNADET with the GP. a, b Histopathological pictures show low- to high-grade adenoma with a villous or papillary structure. c The tumor shows gastric foveolar-like differentiation at the surface area. $\mathbf{d}$ The tumor also shows gastric pyloric gland-like differentiation. e-h IHC for phe-

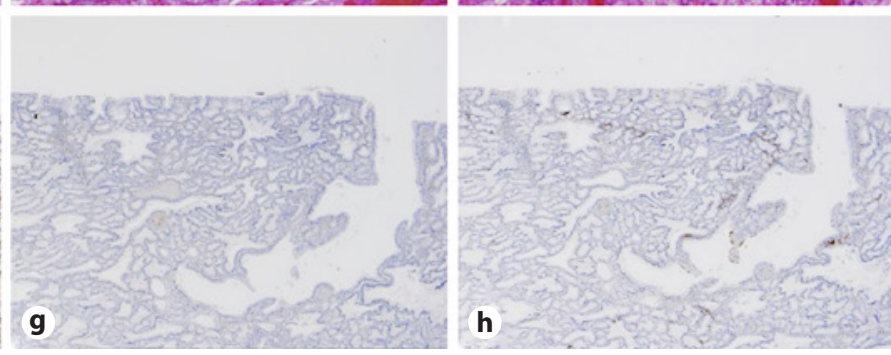

notypes shows positivity for MUC5AC (e) and MUC6 (f) and negativity for MUC2 $(\mathbf{g})$ and CD10 (h), compatible with the gastric mucin phenotype. GP, gastric phenotype; IHC, immunohistochemistry; SNADET, superficial non-ampullary duodenal epithelial tumor.
Fig. 3. Endoscopic findings of a SNADET with the IP. a, b WLI shows a whitish to isochromatic flat elevated lesion in the second portion of the duodenum. c, $\mathbf{d}$ M-NBI reveals WOS (red arrow) and light blue crest (black arrow). SNADET, superficial non-ampullary duodenal epithelial tumor; IP, intestinal phenotype; WLI, white-light imaging; M-NBI, magnifying endoscopy with narrow-band imaging; WOS, white opaque substance.
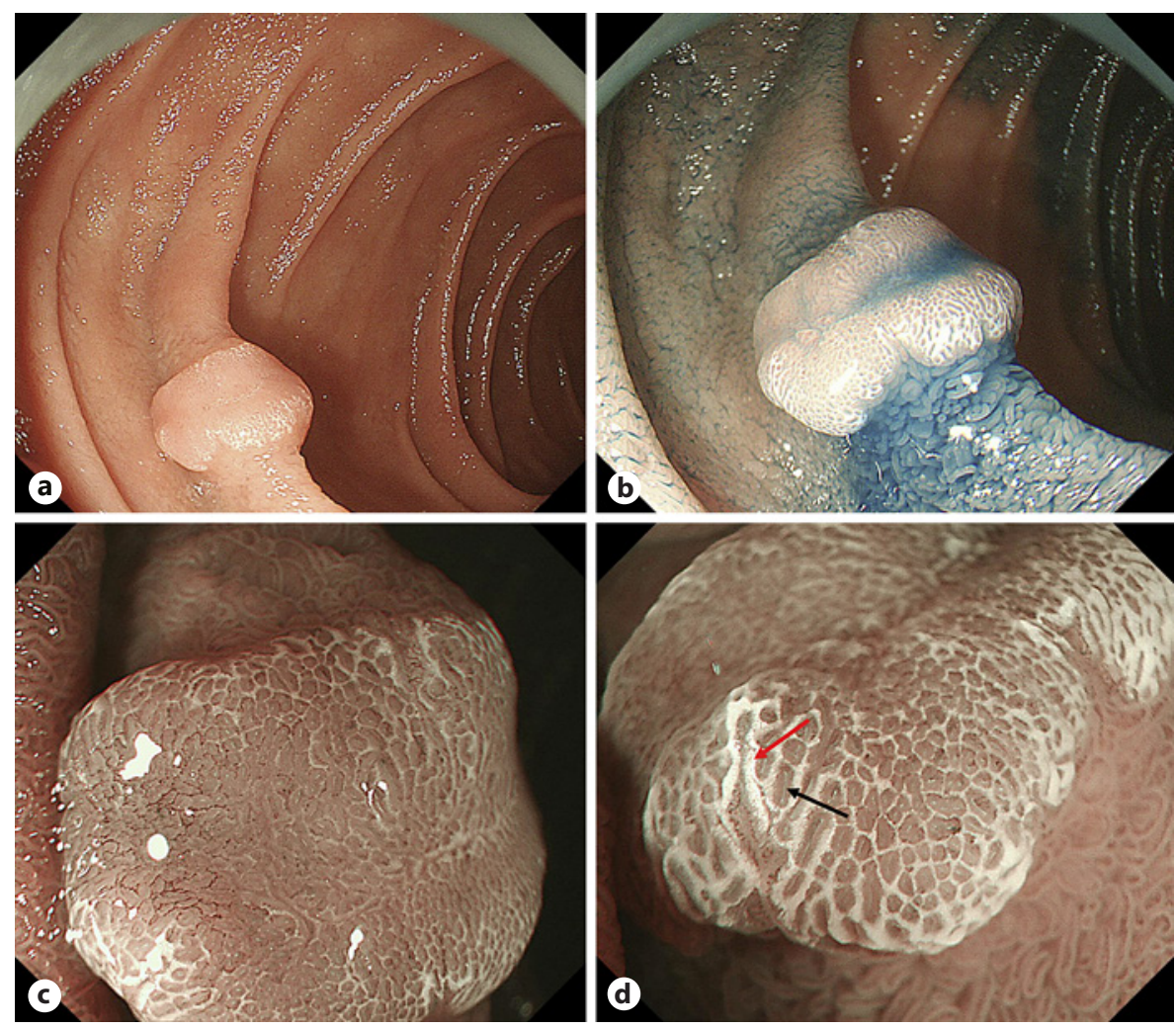

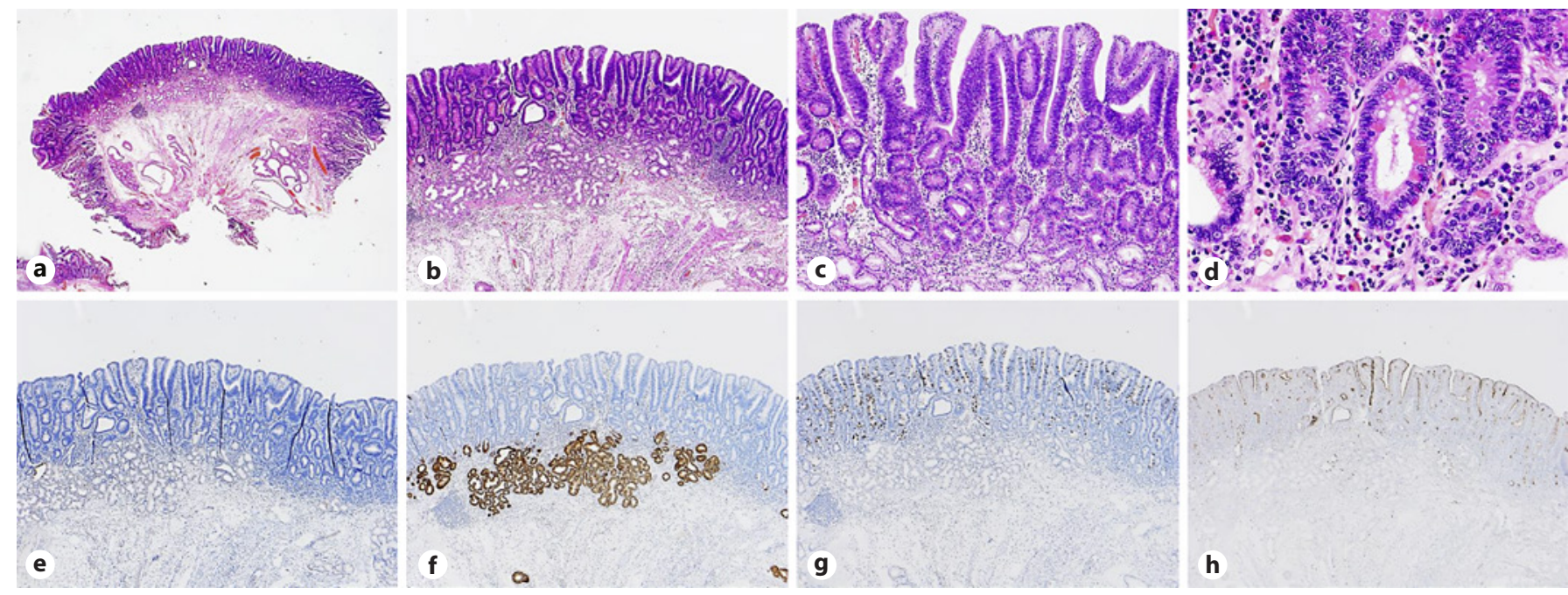

Fig. 4. Histopathological findings of a SNADET with the IP. a, b Histopathological pictures showing low-grade tubular adenoma. c, d The tumor contains goblet cells, Paneth cells, and brush border. e-h IHC for phenotypes shows negativity for MUC5AC

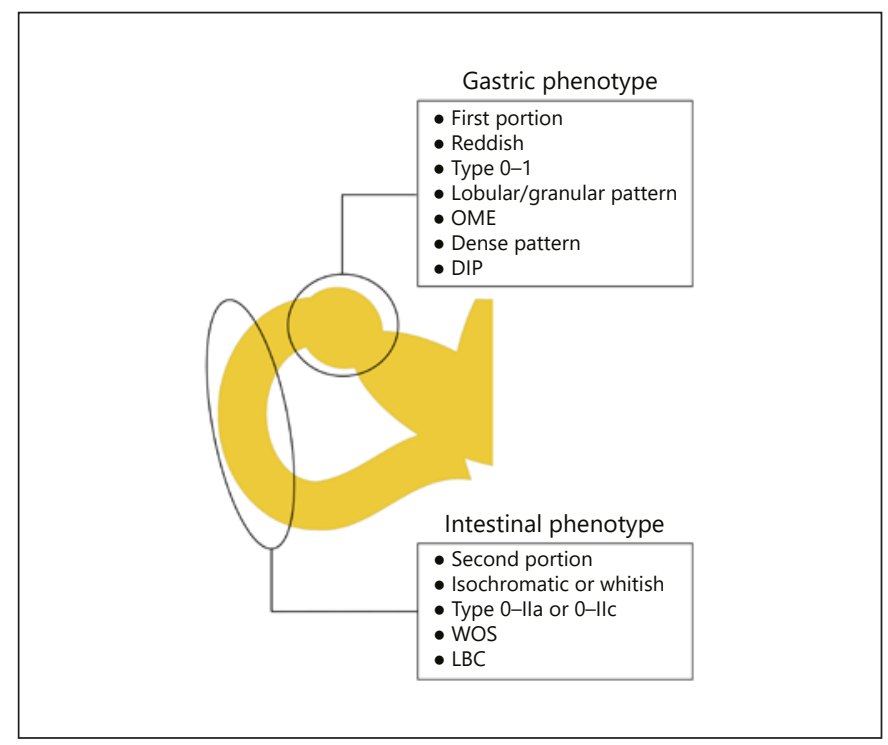

Fig. 5. Summary of the specific findings of the GP and IP of SNADETs. SNADETs, superficial non-ampullary duodenal epithelial tumors; GP, gastric phenotype; IP, intestinal phenotype; OME, oval-shaped marginal epithelium; DIP, dilatation of the intervening part; WOS, white opaque substance; LBC, light blue crest.

formed, WOS was observed in 55.6\% (10/18) of reddish lesions and in 90\% (27/30) of isochromatic or white lesions. Then, such difference in coloration seems to be derived from the distribution of WOS. (e) and MUC6 (f) and positivity for MUC2 (g) and CD10 (h), compatible with the intestinal mucin phenotype. SNADET, superficial non-ampullary duodenal epithelial tumor; IP, intestinal phenotype; IHC, immunohistochemistry.
In the WHO classification, adenoma lesions of the GP are divided into the foveolar type and the pyloric gland type [24]. A few studies reported that adenoma of the GP which predominantly differentiates into the foveolar epithelium could show a villous or papillary structure in the tumor surface area $[8,25]$. Thus, we focused on and analyzed several endoscopic features, such as lobular/granular pattern, OME, dense pattern, and DIP, which reflect villous or papillary structure. These endoscopic findings were observed significantly more frequently in the GP than in the IP group and may reflect histopathological gastric foveolar differentiation at the surface area.

WOS is a substance in the superficial area of a gastric neoplasia and results from the accumulation of lipid droplets with adipophilin $[20,26]$. Moreover, WOS is considered as an indicator of IP [27, 28]. LBC, a bluewhitish patchy area observable by M-NBI on the gastric epithelial surface, has also been thought to be suggestive of gastric intestinal metaplasia, and correlated with CD10-positive cells [19]. In our study, WOS and LBC were significantly more frequently observed in the IP group than in the GP group. From the results of our endoscopic findings, lobular/granular pattern, OME, dense pattern, and DIP were characteristic of the GP, while WOS and LBC were characteristic of the IP. It is suggested that these endoscopic findings make it possible to diagnose mucin phenotypes of SNADETs. 
Interestingly, 2 cases with the GP were positive for WOS, and both the cases were diagnosed as having adenocarcinoma histopathologically. Ueyama et al. [29] reported a case of a WOS-positive gastric hyperplastic polyp with dysplasia and concluded that the presence of WOS suggests neoplastic transformation of the gastric hyperplastic polyp. Moreover, several studies reported that the expression of adipophilin is an indicator of poor prognosis in tumors of other organs [30-32]. Therefore, it is suggested that SNADETs with the GP are positive for WOS when transforming to cancer even if there are no components of the IP. However, it is necessary to study the association between the grade of malignancy and the presence of WOS among SNADETs with the GP because the presence of WOS could be affected by several factors such as food or $\mathrm{pH}$ environment [33].

Several studies reported that gastric-type SNADETs show more aggressive biological behavior than intestinaltype SNADETs [6-9]. Similar to these studies, most cases of the GP $(9 / 11,81.8 \%)$ were diagnosed as category $4 / 5$ in our study. When considering the indication of endoscopic resection, preoperative endoscopic diagnosis is important because the accuracy of duodenal biopsy sampling is relatively low [5]. Goda et al. [2] reported that in SNADETs, diameter $>5 \mathrm{~mm}$ and red color were risk factors for high-grade dysplasia and superficial adenocarcinoma. Similar to those results, our study showed that reddish lesion and larger size of SNADETs were more frequently observed in category $4 / 5$ than in category 3 (see online suppl. Table 1; for all online suppl. material, see www. karger.com/doi/10.1159/000508040). As for endoscopic preoperative diagnosis, several studies reported that MNBI findings were useful for the evaluation of the histological grade in SNDAETs [10, 11, 13]. In our study, both irregular MVP and irregular MSP as evaluated by magnifying endoscopy simple diagnostic algorithm for early gastric cancer (MESDA-G) [18] were significantly more frequently observed in category 4/5 than in category 3 (online suppl. Table 1). Therefore, not only preoperative mucin phenotypic diagnosis but also the M-NBI diagnostic system may be useful for preoperative diagnosis of histological grade.

The limitations of the present study include its singlecenter nature and retrospective study design. In the future, a prospective multicenter comparative study that analyzes inter-rater agreements should be conducted.

In conclusion, endoscopic and clinicopathological features of the GP of SNADETs were as follows: located in the first portion of the duodenum, reddish color, type 0-I, lobular/granular pattern, OME, dense pattern, DIP, and high frequency of category 4/5 in the Vienna classification. On the other hand, endoscopic and clinicopathological features of the IP were as follows: located in the second portion of the duodenum, isochromatic or whitish color, type 0 -IIa or 0 -IIc, WOS, LBC, and high frequency of category 3 in the Vienna classification. These features are useful to distinguish the mucin phenotypes of SNADETs and may be helpful for preoperative diagnosis.

\section{Acknowledgements}

The authors thank Isao Kurahayashi, Noriko Sasahara, and Satomi Saito for their technical assistance and advice.

\section{Statement of Ethics}

This study was reviewed and approved by the Institutional Review Board of Juntendo University School of Medicine (approval number: \#2018292). Patients were not required to give consent for the study because the analysis used anonymous clinical data that were obtained after each patient agreed to treatment by verbal consent. Individuals cannot be identified from the data presented.

\section{Disclosure Statement}

All authors have no financial relationships relevant to this publication.

\section{Funding Sources}

This study was not funded.

\section{Author Contributions}

Yoichi Akazawa and Hiroya Ueyama conceived and designed the study and wrote, edited, and reviewed the manuscript. Sho Tsuyama performed histopathological studies. Takashi Hashimoto and Natsumi Tomita gathered patient clinical information. Motohiko Kato provided a lot of advice about the endoscopic and pathological classification of duodenal tumors in this study. Isao Kurahayashi, Noriko Sasahara, and Satomi Saito provided histological and immunohistochemical technical assistance. All authors gave final approval for publication. Hiroya Ueyama, Yoshiaki Kajiyama, Akihito Nagahara, and Takashi Yao take full responsibility for the work as a whole, including the study design, access to data, and the decision to submit and publish the manuscript. 


\section{References}

1 Endo M, Abiko Y, Oana S, Kudara N, Chiba T, Suzuki K, et al. Usefulness of endoscopic treatment for duodenal adenoma. Dig Endosc. 2010;22(4):360-5.

2 Goda K, Kikuchi D, Yamamoto Y, Takimoto K, Kakushima N, Morita Y, et al. Endoscopic diagnosis of superficial non-ampullary duodenal epithelial tumors in Japan: multicenter case series. Dig Endosc. 2014;26(Suppl 2):23-

3 Inoue T, Uedo N, Yamashina T, Yamamoto S, Hanaoka N, Takeuchi Y, et al. Delayed perforation: a hazardous complication of endoscopic resection for non-ampullary duodenal neoplasm. Dig Endosc. 2014;26(2):220-7.

4 Kakushima N, Kanemoto H, Tanaka M, Takizawa K, Ono H. Treatment for superficial non-ampullary duodenal epithelial tumors. World J Gastroenterol. 2014;20(35):12501-8.

5 Kinoshita S, Nishizawa T, Ochiai Y, Uraoka T, Akimoto T, Fujimoto A, et al. Accuracy of biopsy for the preoperative diagnosis of superficial nonampullary duodenal adenocarcinoma. Gastrointest Endosc. 2017;86(2):32932.

6 Ushiku T, Arnason T, Fukayama M, Lauwers GY. Extra-ampullary duodenal adenocarcinoma. Am J Surg Pathol. 2014;38(11):148493.

7 Hida R, Yamamoto H, Hirahashi M, Kumagai R, Nishiyama K, Gi T, et al. Duodenal neoplasms of gastric phenotype: an immunohistochemical and genetic study with a practical approach to the classification. Am J Surg Pathol. 2017;41(3):343-53.

8 Mitsuishi T, Hamatani S, Hirooka S, Fukasawa N, Aizawa D, Hara Y, et al. Clinicopathological characteristics of duodenal epithelial neoplasms: focus on tumors with a gastric mucin phenotype (pyloric gland-type tumors). PloS One. 2017;12(4):e0174985.

9 Toba T, Inoshita N, Kaise M, Nomura K, Kuribayashi Y, Tanaka M, et al. Clinicopathological features of superficial non-ampurally duodenal epithelial tumor; gastric phenotype of histology correlates to higher malignant potency. J Gastroenterol. 2018;53(1):64-70.

10 Yoshimura N, Goda K, Tajiri H, Ikegami M, Nakayoshi T, Kaise M. Endoscopic features of nonampullary duodenal tumors with narrow-band imaging. Hepatogastroenterology. 2010;57(99-100):462-7.

11 Kikuchi D, Hoteya S, Iizuka T, Kimura R, Kaise M. Diagnostic algorithm of magnifying endoscopy with narrow band imaging for superficial non-ampullary duodenal epithelial tumors. Dig Endosc. 2014;26(Suppl 2):16-22.
12 Tsuji S, Doyama H, Tsuji K, Tsuyama S, Tominaga $\mathrm{K}$, Yoshida N, et al. Preoperative endoscopic diagnosis of superficial non-ampullary duodenal epithelial tumors, including magnifying endoscopy. World J Gastroenterol. 2015;21(41):11832-41.

13 Mizumoto T, Sanomura Y, Tanaka S, Kuroki K, Kurihara M, Yoshifuku Y, et al. Clinical usefulness of magnifying endoscopy for nonampullary duodenal tumors. Endosc Int Open. 2017;5(4):E297-302.

14 Toya Y, Endo M, Akasaka R, Urushikubo J, Gonai T, Asakura K, et al. Clinicopathological features and magnifying chromoendoscopic findings of non-ampullary duodenal epithelial tumors. Digestion. 2018;97(3):219-27.

15 Dixon MF. Gastrointestinal epithelial neoplasia: vienna revisited. Gut. 2002;51(1):130-1.

16 Participants in the Paris Workshop. The Paris endoscopic classification of superficial neoplastic lesions: esophagus, stomach, and colon. Gastrointest Endosc. 2003;58(6):S3-43.

17 Yao K, Anagnostopoulos GK, Ragunath K. Magnifying endoscopy for diagnosing and delineating early gastric cancer. Endoscopy. 2009;41(5):462-7.

18 Muto M, Yao K, Kaise M, Kato M, Uedo N, Yagi K, et al. Magnifying endoscopy simple diagnostic algorithm for early gastric cancer (MESDA-G). Dig Endosc. 2016;28(4):37993.

19 Uedo N, Ishihara R, Iishi H, Yamamoto S, Yamamoto S, Yamada T, et al. A new method of diagnosing gastric intestinal metaplasia: narrow-band imaging with magnifying endoscopy. Endoscopy. 2006;38(8):819-24.

20 Yao K, Iwashita A, Tanabe H, Nishimata N, Nagahama T, Maki S, et al. White opaque substance within superficial elevated gastric neoplasia as visualized by magnification endoscopy with narrow-band imaging: a new optical sign for differentiating between adenoma and carcinoma. Gastrointest Endosc. 2008; 68(3):574-80

21 Wada Y, Kudo SE, Kashida H, Ikehara N, Inoue $\mathrm{H}$, Yamamura F, et al. Diagnosis of colorectal lesions with the magnifying narrow-band imaging system. Gastrointest Endosc. 2009;70(3);522-31.

22 Kanda Y. Investigation of the freely available easy-to-use software "EZR" for medical statistics. Bone Marrow Transplant. 2013;48(3): 452-8.

23 Hijikata K, Nemoto T, Igarashi Y, Shibuya K. Extra-ampullary duodenal adenoma: a clinicopathological study. Histopathology. 2017; 71(2):200-7.
24 Bosman FT, Carneiro F, Hruban RH, Theise ND. WHO classification of tumours of the digestive system. 4th ed. France: IARC; 2010.

25 Kushima R, Rüthlein HJ, Stolte M, Bamba M, Hattori T, Borchard F. "Pyloric gland-type adenoma" arising in heterotopic gastric mucosa of the duodenum, with dysplastic progression of the gastric type. Virchows Arch. 1999;435(4):452-7.

26 Ueo T, Yonemasu H, Yada N, Yano S, Ishida $\mathrm{T}$, Urabe $\mathrm{M}$, et al. White opaque substance represents an intracytoplasmic accumulation of lipid droplets: immunohistochemical and immunoelectron microscopic investigation of 26 cases. Dig Endosc. 2013;25(2):147-55.

27 Yao K, Iwashita A, Nambu M, Tanabe H, Nagahama T, Maki S, et al. Nature of white opaque substance in gastric epithelial neoplasia as visualized by magnifying endoscopy with narrow-band imaging. Dig Endosc. 2012;24(6):419-25

28 Ueo T, Yonemasu H, Yao K, Ishida T, Togo $\mathrm{K}$, Yanai $\mathrm{Y}$, et al. Histologic differentiation and mucin phenotype in white opaque substance-positive gastric neoplasias. Endosc Int Open. 2015;3(6):E597-604.

29 Ueyama H, Matsumoto K, Nagahara A, Gushima R, Hayashi T, Yao T, et al. A white opaque substance-positive gastric hyperplastic polyp with dysplasia. World J Gastroenterol. 2013;19(26):4262-6.

30 Yao M, Huang Y, Shioi K, Hattori K, Murakami T, Nakaigawa N, et al. Expression of adipose differentiation-related protein: a predictor of cancer-specific survival in clear cell renal carcinoma. Clin Cancer Res. 2007; 13(1):152-60.

31 Matsubara J, Honda K, Ono M, Sekine S, Tanaka Y, Kobayashi M, et al. Identification of adipophilin as a potential plasma biomarker for colorectal cancer using label-free quantitative mass spectrometry and protein microarray. Cancer Epidemiol Biomarkers Prev. 2011;20(10):2195-203.

32 Hashimoto Y, Ishida M, Ryota H, Yamamoto T, Kosaka H, Hirooka S, et al. Adipophilin expression is an indicator of poor prognosis in patients with pancreatic ductal adenocarcinoma: an immunohistochemical analysis. Pancreatology. 2019;19(3):443-8.

33 Togo K, Ueo T, Yao K, Wada K, Honda H, Inoue $\mathrm{S}$, et al. White opaque substance visualized by magnifying narrow-band imaging is associated with intragastric acid conditions. Endosc Int Open. 2018;6(7):E830-37. 